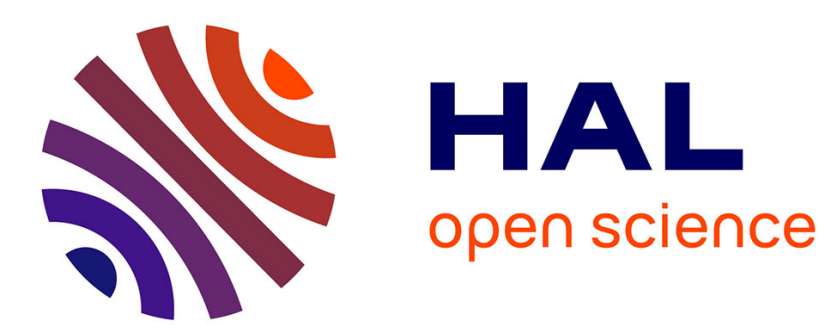

\title{
Pseudofinite Difference Fields
}

Tingxiang Zou

\section{- To cite this version:}

Tingxiang Zou. Pseudofinite Difference Fields. 2018. hal-01831524

\section{HAL Id: hal-01831524 \\ https://hal.science/hal-01831524}

Preprint submitted on 6 Jul 2018

HAL is a multi-disciplinary open access archive for the deposit and dissemination of scientific research documents, whether they are published or not. The documents may come from teaching and research institutions in France or abroad, or from public or private research centers.
L'archive ouverte pluridisciplinaire HAL, est destinée au dépôt et à la diffusion de documents scientifiques de niveau recherche, publiés ou non, émanant des établissements d'enseignement et de recherche français ou étrangers, des laboratoires publics ou privés. 


\title{
Pseudofinite Difference Fields
}

\author{
Tingxiang Zou*(zou@math.univ-lyon1.fr)
}

\begin{abstract}
We study a family of pseudofinite difference fields in this paper. Their theories have the strict order property and TP2. But the definable sets of these structures still have some nice properties. In particular, we show that the coarse dimension of the definable sets is definable and integer-valued.
\end{abstract}

\section{Introduction}

The class of various expansions of fields is one of the key objects of study in model theory. Examples are differentially closed fields, Henselian valued fields, algebraically closed fields with a generic automorphism, etc. There are lots of natural examples of such structures that are intensively investigated in other areas of mathematics, while the model theories of them often extends well-known results to a wider context and sometimes, model theoretic techniques can help to discover new phenomenons.

We will consider expansions of pseudofinite fields with a distinguished automorphism. The model theory of pseudofinite fields has been initiated by J. Ax in [1] and subsequently developed in [7], [6], [9]. On the other hand, the model theory of fields with a distinguished automorphism has also been investigated. The best understood one is possibly ACFA: the theory of algebraically closed fields with a generic automorphism, developed notably in [4], [5]. It is the model companion of the theory of difference fields and, interestingly, the fixed field of any model of ACFA is a pseudofinite field. Based on these, one might expect a theory of pseudofinite difference fields which is a mixture of PSF (the theory of pseudofinite fields) and ACFA.

M. Ryten has studied a specific class of pseudofinite difference fields with the motivation of understanding the asymptotic behaviour of Suzuki groups and Ree groups. In [12], he showed that given any prime $p$ and a pair of coprime numbers $m, n>1$, the class $\left\{\left(\mathbb{F}_{p^{k_{p} \cdot m+n}}\right.\right.$, Frob $\left.\left._{p^{k_{p}}}\right): k_{p} \in \mathbb{N}\right\}$ is a one-dimensional asymptotic class. He also gave a recursive axiomatization of asymptotic theories of such structures: $P S F_{(m, n, p)}$. In a sense, $P S F_{(m, n, p)}$ is a mixture of PSF and ACFA. In fact, any model of $P S F_{(m, n, p)}$ can be obtained as a definable substructure of some model of $\mathrm{ACFA}^{1}$, and the onedimensional asymptotic class result is based on the uniform estimate of the number of solutions of definable sets of finite $\sigma$-degree in some model of ACFA in [11].

However $P S F_{(m, n, p)}$ is a bit restricted in the sense that in no model of $P S F_{(m, n, p)}$ there are transformally transcendental elements, elements that satisfy no non-trivial

\footnotetext{
*This author is supported by the China Scholarship Council and partially supported by ValCoMo (ANR-13-BS01-0006).

${ }^{1}$ See [12, Lemma 3.3.6].
} 
difference polynomial. Our aim in this paper is to study a class of pseudofinite difference fields with transformally transcendental elements.

Another class of closely related structures is the class of pairs of pseudofinite fields, as the fixed field of a pseudofinite difference field is finite or pseudofinite. As noticed by Macintyre and Cherlin, there are pairs of pseudofinite fields whose theory is not decidable. This wild phenomenon also occurs in the structures that we study, but we also gain some tameness properties of definable sets, see Theorem 9. We think it is possible to have pseudofinite difference fields with transformally transcendental elements whose theory is still decidable. But it is not clear what kind of theories they are.

Acknowledgement: the author wants to thank her supervisor Frank Wagner for initiating this interesting project and contributing lots of valuables ideas. She also wants to thank Zoé Chatzidakis for answering various questions about pseudofinite fields and ACFA, and Dario Garcia for suggestions of corrections on the previous version of this paper.

\section{Pseudofinite coarse dimension}

We begin with some preliminaries of difference fields.

Definition 1. A difference field is a field $(F,+, \cdot, 0,1)$ together with a field automorphism $\sigma$ which is surjective.

The language of difference rings $\mathcal{L}_{\sigma}$ is the language of rings augmented by a unary function symbol $\sigma$.

Definition 2. We fix an ambient difference field $L$.

- Let $A$ be a subset. We denote by $A_{\sigma}$ to be the smallest difference subfield containing $A$ and closed under $\sigma$ and $\sigma^{-1}$.

- Let $E$ be a difference subfield and $a$ be a tuple. The $\sigma$-degree, $\operatorname{deg}_{\sigma}(a / E)$, is the transcendence degree of $(E, a)_{\sigma}$ over $E$.

- Let $E$ be a difference subfield. If there is no non-zero difference polynomial over $E$ vanishing on $a$, then we say $a$ is transformally transcendental over $E$ if $a$ is an element in $L$ and $a$ is transformally independent over $E$ if $a$ is a tuple in $L$.

- Let $E$ be a difference subfield and $a$ be a tuple. The transformal transcendence degree of $a$ over $E$ is defined as the maximal length of a transformally independent subtuple of $a$ over $E$.

We now give the definition of pseudofinite coarse dimension.

Definition 3. Let $M$ be a pseudofinite structure over some non-principal ultrafilter $\mathcal{U}$ and $\mathbb{R}^{*}$ be the ultrapower of $\mathbb{R}$ along $\mathcal{U}$. Then any pseudofinite set $D \subseteq M^{n}$ has a non-standard cardinality $|D| \in \mathbb{R}^{*}$. Let $\alpha \in \mathbb{R}^{*}$.

- The coarse dimension on $M$ normalised by $\alpha$, denoted $\boldsymbol{\delta}_{\alpha}$, is a function from definable sets of $M$ to $\mathbb{R}^{\geq 0} \cup\{\infty\}$, defined as

$$
\boldsymbol{\delta}_{\alpha}(A):=\operatorname{st}\left(\frac{\log |A|}{\alpha}\right),
$$


for $A \subseteq M^{n}$ definable. When $\alpha:=\log |X|$ for some pseudofinite set $X$, we also write $\boldsymbol{\delta}_{\alpha}$ as $\boldsymbol{\delta}_{X}$.

- We say $\boldsymbol{\delta}_{\alpha}$ is continuous if for any $\emptyset$-definable formula $\phi(x, y)$, for any $r_{1}<r_{2} \in \mathbb{R}$, there is some $\emptyset$-definable set $D$ with

$$
\left\{a \in M^{|y|}: \delta_{\alpha}\left(\phi\left(M^{|x|}, a\right)\right) \leq r_{1}\right\} \subseteq D \subseteq\left\{a \in M^{|y|}: \boldsymbol{\delta}_{\alpha}\left(\phi\left(M^{|x|}, a\right)\right)<r_{2}\right\} .
$$

- We say $\boldsymbol{\delta}_{\alpha}$ is definable if $\boldsymbol{\delta}_{\alpha}$ is continuous and the set $\left\{\boldsymbol{\delta}_{\alpha}\left(\phi\left(M^{|x|}, a\right)\right): a \in M^{|\bar{y}|}\right\}$ is finite for any $\emptyset$-definable formula $\phi(x, y)$. By compactness, it is equivalent to the following: for any $\emptyset$-definable formula $\phi(x, y)$ and $a \in M^{|y|}$, there is $\xi(y) \in \operatorname{tp}(a)$ such that

$$
M \models \xi(b) \text { if and only if } \boldsymbol{\delta}_{\alpha}\left(\phi\left(M^{|x|}, b\right)\right)=\boldsymbol{\delta}_{\alpha}\left(\phi\left(M^{|x|}, a\right)\right)
$$

Definition 4. Let $M$ be a pseudofinite structure and $\alpha \in \mathbb{R}^{*}$. Let $a$ be a tuple in $M$ and $A \subseteq M$. Define

$$
\boldsymbol{\delta}_{\alpha}(a / A):=\inf \left\{\boldsymbol{\delta}_{\alpha}\left(\varphi\left(M^{|x|}\right)\right), \varphi(x) \in \operatorname{tp}(a / A)\right\} .
$$

Fact 5. [8, Lemma 2.10] If $\boldsymbol{\delta}_{\alpha}$ is continuous, then $\boldsymbol{\delta}_{\alpha}$ is additive, i.e., for any $a, b, A \subseteq M$ we have $\boldsymbol{\delta}_{\alpha}(a, b / A)=\boldsymbol{\delta}_{\alpha}(a / A, b)+\boldsymbol{\delta}_{\alpha}(b / A)$.

Remark: There is always a way to make $\boldsymbol{\delta}_{\alpha}$ continuous by expanding the language of the structure $M$. However, this might add new definable sets to $M$, which could be an inconvenience.

The following fact is a well-known result in the class of finite fields, which gives a uniform estimate of number of solutions of definable sets in all finite fields. Our main result will be based on it.

Fact 6. [6] Let $\mathcal{L}$ be the language of rings. For every formula $\varphi(x, y) \in \mathcal{L}$ with $|x|=$ $n,|y|=m$ there are a constant $C_{\varphi}>0$ and a finite set $D_{\varphi} \subset\{0, \ldots, n\} \times \mathbb{R}^{>0}$ such that the following holds:

For any finite field $\mathbb{F}_{q}$ and $a \in\left(\mathbb{F}_{q}\right)^{m}$, if $\varphi\left(\left(\mathbb{F}_{q}\right)^{n}, a\right) \neq \emptyset$, then there is some $(d, \mu) \in D$ such that

$$
|| \varphi\left(\left(\mathbb{F}_{q}\right)^{n}, a\right)\left|-\mu \cdot q^{d}\right| \leq C_{\varphi} \cdot q^{d-\frac{1}{2}} .
$$

Now we start to define a special class of pseudofinite difference fields and study the model theoretic properties of them.

Definition 7. Let $\mathcal{L}_{\sigma}$ be the language of difference rings. Let $\varphi(x, y)$ be a formula defined in $\mathcal{L}_{\sigma}$ without parameters. For any prime $p$, define $\varphi_{p}(x, y)$ as the result of replacing all occurrence of $\sigma(t)$ by $t^{p}$. Clearly, $\varphi_{p}(x, y)$ is a formula in the language of rings $\mathcal{L}$.

Let $\mathbb{P}$ be the set of all primes. For any formula $\varphi(x, y)$ in $\mathcal{L}_{\sigma}$ and $p \in \mathbb{P}$, consider $\varphi_{p}(x, y) \in \mathcal{L}$. There are $C_{\varphi_{p}}$ and the finite set $D_{\varphi_{p}}$ as stated in Fact 6 . Let

$$
E_{\varphi_{p}}:=\bigcup_{0 \leq d \leq|x|}\left\{\mu:(d, \mu) \in D_{\varphi_{p}}\right\}
$$


Define

$$
N_{\varphi(x, y)}^{p}:=\max \left\{\mu, \frac{1}{\mu}, 2 \log _{p} \frac{2 C_{\varphi_{p}}}{\mu}: \mu \in E_{\varphi_{p}}\right\}
$$

Let

$$
f(\ell, p):=\max \left\{N_{\varphi(x, y)}^{p}:|\varphi(x, y)| \leq \ell\right\} .
$$

Definition 8. Define the family $\mathcal{S}$ of pseudofinite difference fields as $\mathcal{S}:=\left\{\prod_{p \in \mathbb{P}}\left(\mathbb{F}_{p^{k_{p}}}, \operatorname{Frob}_{p}\right) / \mathcal{U}: k_{p} \geq f(p, p)\right.$ for all $p \in \mathbb{P}, \mathcal{U}$ a non-principal ultrafilter $\}$.

Theorem 9. Let $(F$, Frob $):=\prod_{p \in \mathbb{P}}\left(\mathbb{F}_{p^{k_{p}}}\right.$, Frob $\left._{p}\right) / \mathcal{U} \in \mathcal{S}$. Then $\boldsymbol{\delta}_{F}$, the pseudofinite coarse dimension normalised by $|F|$, is integer-valued on all $\mathcal{L}_{\sigma}$-definable set .

Proof. Let $\varphi(x, y)$ be an $\mathcal{L}_{\sigma^{-}}$formula. Consider a parameter $a=\left(a_{p}\right)_{p \in \mathbb{P}} / \mathcal{U} \in F^{|y|}$. For any $p \in \mathbb{P}$, we know that there are $\left(d_{k_{p}}, \mu_{k_{p}}\right) \in\{0, \ldots,|x|\} \times \mathbb{R}^{>0}$ and $C_{\varphi_{p}} \geq 0$ such that for $a_{p} \in\left(\mathbb{F}_{p^{k_{p}}}\right)^{|y|}$, we have

$$
|| \varphi_{p}\left(\left(\mathbb{F}_{p^{k_{p}}}\right)^{|x|}, a_{p}\right)\left|-\mu_{k_{p}} \cdot p^{k_{p} \cdot d_{k_{p}}}\right| \leq C_{\varphi_{p}} \cdot p^{k_{p}\left(d_{k_{p}}-\frac{1}{2}\right)} .
$$

We say that $\varphi_{p}\left(x, a_{p}\right)$ has dimension $d_{k_{p}}$ in $\mathbb{F}_{p^{k_{p}}}$. As $d_{k_{p}} \leq|x|$, there is exactly one $d \in\{0, \ldots,|x|\}$ with $\left\{p \in \mathbb{P}: \varphi_{p}\left(x, a_{p}\right)\right.$ has dimension $d$ in $\left.\mathbb{F}_{p^{k_{p}}}\right\} \in \mathcal{U}$. We claim that $\boldsymbol{\delta}_{F}\left(\varphi\left(F^{|x|}, a\right)\right)=d$.

Proof of the claim: Note that for any $p \in \mathbb{P}$ and $c \in\left(\mathbb{F}_{p_{p}}\right)^{|x|}$, we have

$$
\mathbb{F}_{p^{k_{p}}} \models \varphi_{p}\left(c, a_{p}\right) \text { if and only if }\left(\mathbb{F}_{p^{k_{p}}}, \text { Frob }_{p}\right) \models \varphi\left(c, a_{p}\right) .
$$

Let $I=\left\{p \in \mathbb{P}: p>|\varphi(x, y)|\right.$ and $\varphi_{p}\left(x, a_{p}\right)$ has dimension $d$ in $\left.\mathbb{F}_{p^{k_{p}}}\right\}$. Clearly, $I \in \mathcal{U}$. Then for any $p \in I$,

$$
|| \varphi_{p}\left(\left(\mathbb{F}_{p^{k_{p}}}\right)^{|x|}, a_{p}\right)\left|-\mu_{k_{p}} \cdot p^{k_{p} \cdot d}\right| \leq C_{\varphi_{p}} \cdot p^{k_{p}\left(d-\frac{1}{2}\right)},
$$

and $k_{p} \geq f(p, p) \geq \max \left\{\mu_{k_{p}}, \frac{1}{\mu_{k_{p}}}, 2 \log _{p} \frac{2 C_{\varphi_{p}}}{\mu_{k_{p}}}\right\}$.

As $k_{p} \geq 2 \log _{p} \frac{2 C_{\varphi_{p}}}{\mu_{k_{p}}}$, we get

$$
C_{\varphi_{p}} \cdot p^{k_{p}\left(d-\frac{1}{2}\right)} \leq \frac{1}{2} \mu_{k_{p}} \cdot p^{k_{p} \cdot d}
$$

Therefore,

$$
\frac{1}{2} \mu_{k_{p}} \cdot p^{k_{p} \cdot d} \leq\left|\varphi_{p}\left(\left(\mathbb{F}_{p^{k_{p}}}\right)^{|x|}, a_{p}\right)\right| \leq \frac{3}{2} \mu_{k_{p}} \cdot p^{k_{p} \cdot d} .
$$

Furthermore, by the definition of $k_{p}$, we have $\frac{1}{k_{p}}<\mu_{k_{p}}<k_{p}$. Hence,

$$
\frac{1}{2 k_{p}} \cdot p^{k_{p} \cdot d} \leq\left|\varphi_{p}\left(\left(\mathbb{F}_{p^{k_{p}}}\right)^{|x|}, a_{p}\right)\right| \leq 2 k_{p} \cdot p^{k_{p} \cdot d}
$$

This implies

$$
d-\frac{\log \left(2 k_{p}\right)}{k_{p} \cdot \log p} \leq \frac{\log \left|\varphi_{p}\left(\left(\mathbb{F}_{p^{k_{p}}}\right)^{|x|}, a_{p}\right)\right|}{\log \left(p^{k_{p}}\right)} \leq d+\frac{\log \left(2 k_{p}\right)}{k_{p} \cdot \log p} .
$$


Obviously, we have

$$
\lim _{p \rightarrow \infty, p \in I} \frac{\log \left|\varphi_{p}\left(\left(\mathbb{F}_{p^{k_{p}}}\right)^{|x|}, a_{p}\right)\right|}{\log \left(p^{k_{p}}\right)}=d .
$$

Therefore, $\boldsymbol{\delta}_{F}\left(\varphi\left(F^{|x|}, a\right)\right)=d$.

Remark: This proof works also for pseudofinite difference fields of characteristic $p>0$, that is, for $\prod_{i \in I}\left(\mathbb{F}_{p^{k_{i}}}\right.$, Frob $\left._{p^{t_{i}}}\right) / \mathcal{U}$ provided $k_{i}>>t_{i}$ for almost all $i$.

In the following, we will show that the coarse dimension $\boldsymbol{\delta}_{F}$ is definable using the field structure. To prove this, we first need a lemma.

Lemma 10. Let $\mathcal{M}$ be a pseudofinite structure in the language $\mathcal{L}_{M}$ and $X$ be a pseudofinite subset of $M$. Let $\varphi(x, y)$ be an $\mathcal{L}_{M}$-formula with $|x|=m$ and $|y|=n$. Suppose there is some $r \in \mathbb{R}^{\geq 0}$ such that for all $b \in M^{m}$ we have $\boldsymbol{\delta}_{X}\left(\varphi\left(M^{n}, b\right)\right)=r$ whenever $\varphi\left(M^{n}, b\right) \neq \emptyset$. Then

$$
\boldsymbol{\delta}_{X}\left(\varphi\left(M^{n+m}\right)\right)=r+\boldsymbol{\delta}_{X}\left(\exists x \varphi\left(x, M^{m}\right)\right) .
$$

Proof. Suppose $(M, X)=\prod_{i \in I}\left(M_{i}, X_{i}\right) / \mathcal{U}$ for some ultrafilter $\mathcal{U}$ on an index set $I$ and $X_{i} \subseteq M_{i}$ finite sets. For each $i \in I$ pick $b_{i}^{\text {max }}$ and $b_{i}^{\text {min }}$ in $\left(M_{i}\right)^{m}$ such that $\left|\varphi\left(\left(M_{i}\right)^{n}, b_{i}^{\max }\right)\right|$ is maximal and $\left|\varphi\left(\left(M_{i}\right)^{n}, b_{i}^{\text {min }}\right)\right|$ is minimal non-zero respectively. Clearly, we have

$$
\left|\varphi\left(\left(M_{i}\right)^{n}, b_{i}^{\min }\right)\right| \cdot\left|\exists x \varphi\left(x,\left(M_{i}\right)^{m}\right)\right| \leq\left|\varphi\left(\left(M_{i}\right)^{n+m}\right)\right| \leq \varphi\left(\left(M_{i}\right)^{n}, b_{i}^{\max }\right)|\cdot| \exists x \varphi\left(x,\left(M_{i}\right)^{m}\right) \mid .
$$

Let $b^{\text {max }}:=\left(b_{i}^{\max }\right)_{i \in I} \mathcal{U} \in M$ and $b^{\text {min }}:=\left(b_{i}^{\text {min }}\right)_{i \in I} / \mathcal{U} \in M$ respectively. By assumption, $\boldsymbol{\delta}_{X}\left(\varphi\left(M^{n}, b^{\max }\right)\right)=\boldsymbol{\delta}_{X}\left(\varphi\left(M^{n}, b^{\min }\right)\right)=r$. Therefore, for any $\epsilon>0$, there is some $J \in \mathcal{U}$ such that for all $i \in J$, we have

$$
\left|X_{i}\right|^{r-\epsilon} \leq\left|\varphi\left(\left(M_{i}\right)^{n}, b_{i}^{\min }\right)\right| \leq\left|\varphi\left(\left(M_{i}\right)^{n}, b_{i}^{\max }\right)\right| \leq\left|X_{i}\right|^{r+\epsilon} .
$$

Multiplying each term by $\left|\exists x \varphi\left(x,\left(M_{i}\right)^{m}\right)\right|$ and combining the inequality before, we get

$$
\left|X_{i}\right|^{r-\epsilon} \cdot\left|\exists x \varphi\left(x,\left(M_{i}\right)^{m}\right)\right| \leq \varphi\left(\left(M_{i}\right)^{n+m}\right) \leq\left|X_{i}\right|^{r+\epsilon} \cdot\left|\exists x \varphi\left(x,\left(M_{i}\right)^{m}\right)\right| .
$$

Therefore,

$$
r-\epsilon+\frac{\log \left|\exists x \varphi\left(x,\left(M_{i}\right)^{m}\right)\right|}{\log \left|X_{i}\right|} \leq \frac{\log \left|\varphi\left(\left(M_{i}\right)^{n+m}\right)\right|}{\log \left|X_{i}\right|} \leq r+\epsilon+\frac{\log \left|\exists x \varphi\left(x,\left(M_{i}\right)^{m}\right)\right|}{\log \left|X_{i}\right|} .
$$

By the definition of $\boldsymbol{\delta}_{X}$ we conclude that

$$
r+\epsilon+\boldsymbol{\delta}_{X}\left(\exists x \varphi\left(x, M^{m}\right)\right) \leq \boldsymbol{\delta}_{X}\left(\varphi\left(M^{n+m}\right)\right) \leq r-\epsilon+\boldsymbol{\delta}_{X}\left(\exists x \varphi\left(x, M^{m}\right)\right) .
$$

Since $\epsilon$ is arbitrary, we get the desired result.

Corollary 11. Let $M$ be a pseudofinite structure in the language $\mathcal{L}$ and let $X \subseteq M^{n}$ be a pseudofinite subset. Suppose there is some $r \in \mathbb{N}$ such that for any $\mathcal{L}$-formula $\varphi(x, y)$ with $|x|=1$ over $\emptyset$ and any $b \in M^{|y|}$, we have $\boldsymbol{\delta}_{X}(\varphi(M, b)) \in\{0,1, \ldots, r\}$ and for each $i \leq r$, the set

$$
\left\{b \in M^{|y|}: \boldsymbol{\delta}_{X}(\varphi(M, b))=i\right\}
$$

is $\emptyset$-definable. Then for any formula $\psi(x, y)$ and any tuple $c \in M^{|y|}$, we have

$$
\boldsymbol{\delta}_{X}\left(\psi\left(M^{|x|}, c\right)\right) \in\{0, \ldots,|x| \cdot r\} .
$$

Moreover, $\boldsymbol{\delta}_{X}$ is definable. 
Proof. We use induction on the length of $|x|$. The case $|x|=1$ is given by assumption.

Suppose the conclusion holds for $|x|=n$, we prove it for $|x|=n+1$. Let $\psi\left(x_{0}, \ldots, x_{n}, y\right)$ be a formula with $\left|x_{i}\right|=1$ for $0 \leq i \leq n$. We know that there are $\emptyset$-definable $\theta_{\ell}\left(x_{1}, \ldots, x_{n}, y\right)$ with $\ell \in\{0,1, \ldots, r\}$ which define respectively the sets

$$
\left\{\left(x_{1}, \ldots, x_{n}, y\right) \in M^{n+|y|}: \boldsymbol{\delta}_{M}\left(\psi\left(M, x_{1}, \ldots, x_{n}, y\right)\right)=\ell \text { and } \psi\left(M, x_{1}, \ldots, x_{n}, y\right) \neq \emptyset\right\} .
$$

For any $c \in M^{|y|}$, note that $\psi\left(M^{n+1}, c\right)$ is the disjoint union of

$$
\left\{\psi\left(M^{n+1}, c\right) \wedge \theta_{i}\left(M^{n}, c\right): i \in\{0,1, \ldots, r\}\right\},
$$

and Lemma 10 applies to each of the formulas. Hence,

$\boldsymbol{\delta}_{X}\left(\psi\left(M^{n+1}, c\right) \wedge \theta_{i}\left(M^{n}, c\right)\right)=i+\boldsymbol{\delta}_{X}\left(\exists x_{0}\left(\psi\left(x_{0}, M^{n}, c\right) \wedge \theta_{i}\left(M^{n}, c\right)\right)=i+\boldsymbol{\delta}_{X}\left(\theta_{i}\left(M^{n}, c\right)\right)\right.$.

By induction hypothesis, $\boldsymbol{\delta}_{X}\left(\theta_{i}\left(M^{n}, c\right)\right) \in\{0, \ldots, r \cdot n\}$. Therefore,

$$
\boldsymbol{\delta}_{X}\left(\psi\left(M^{n+1}, c\right)\right)=\max \left\{i+\boldsymbol{\delta}_{X}\left(\theta_{i}\left(M^{n}, c\right)\right): 0 \leq i \leq r\right\} \in\{0, \ldots, r \cdot(n+1)\} .
$$

Again by induction hypotheses, for any $k \in\{0, \ldots, r \cdot n\}$ there are $\emptyset$-definable $\xi_{i}^{k}(y)$ with $i \in\{0, \ldots, r\}$, which define the corresponding sets

$$
\left\{y \in F^{|y|}: \boldsymbol{\delta}_{X}\left(\theta_{i}\left(M^{n}, y\right)\right)=k \text { and } \theta_{i}\left(M^{n}, y\right) \neq \emptyset\right\} .
$$

Then the formula

$$
\bigvee_{0 \leq i \leq r, 0 \leq j \leq r \cdot n, i+j=t} \xi_{i}^{j}(y)
$$

defines the set

$$
\left\{y \in M^{n+1}: \boldsymbol{\delta}_{M}\left(\psi\left(M^{n+1}, y\right)\right)=t \text { and } \psi\left(M^{n+1}, y\right) \neq \emptyset\right\}
$$

for any $t \in\{0, \ldots, r \cdot(n+1)\}$.

Lemma 12. Let $\mathcal{M}=(F,+, \cdot, 0,1, \ldots)$ be a pseudofinite field with some extra structures. Let $\boldsymbol{\delta}_{F}$ be the pseudofinite coarse dimension normalised by $|F|$. Suppose for any formula $\varphi(x, y)$ with $|x|=1$ we have $\boldsymbol{\delta}_{F}(\varphi(F, b)) \in\{0,1\}$ for any tuple $b \in F^{|y|}$. Then $\boldsymbol{\delta}_{F}$ is definable and for any formula $\psi(x, y)$ and any tuple $c \in F^{|y|}$, we have $\boldsymbol{\delta}_{F}\left(\psi\left(F^{|x|}, c\right)\right) \in\{0, \ldots,|x|\}$.

Proof. By Corollary 11, we only need to show definability when $|x|=1$.

For any $\psi(x, y)$, let

$$
\theta_{\psi}(y):=\forall z \exists x_{1} \exists x_{2} \exists x_{3} \exists x_{4}\left(\bigwedge_{1 \leq i \leq 4} \psi\left(x_{i}, y\right) \wedge x_{3} \neq x_{4} \wedge z=\left(x_{1}-x_{2}\right) \cdot\left(x_{3}-x_{4}\right)^{-1}\right) .
$$

We claim that $\theta_{\psi}(c)$ if and only if $\boldsymbol{\delta}_{F}(\psi(F, c))=1$ for all $c \in F^{|y|}$. Suppose $\theta_{\psi}(c)$ holds, then clearly there is a surjection from $(\psi(F, c))^{4}$ to $F$. Therefore, $\boldsymbol{\delta}_{F}(\psi(F, c)) \geq \frac{1}{4}$. By assumption, $\boldsymbol{\delta}_{F}(\psi(F, c)) \in\{0,1\}$. Hence, $\boldsymbol{\delta}_{F}(\psi(F, c))=1$. On the other hand, if $\neg \theta_{\psi}(c)$ holds, there is $a \in F$ such that for any $x_{1}, x_{2}, x_{3}, x_{4} \in \psi(F, c)$ we have $a \neq\left(x_{1}-x_{2}\right)\left(x_{3}-\right.$ $\left.x_{4}\right)^{-1}$ whenever $x_{3} \neq x_{4}$. Let $f:(\psi(F, c))^{2} \rightarrow F$ be defined as $f\left(x_{1}, x_{2}\right):=x_{1}+a x_{2}$. Then $f$ is an injection. Therefore, $\boldsymbol{\delta}_{F}(\psi(F, c)) \leq \frac{1}{2}$. We conclude that $\boldsymbol{\delta}_{F}(\psi(F, c))=0$. 
Hence, the set

$$
\left\{c \in F^{|y|}: \delta_{F}(\psi(F, c))=0 \text { and } \psi(F, c) \neq \emptyset\right\}
$$

is defined by $\neg \theta_{\psi}(y) \wedge \exists x \psi(x, y)$. And $\theta_{\psi}(y)$ defines the set

$$
\left\{c \in F^{|y|}: \boldsymbol{\delta}_{F}(\psi(F, y))=1\right\} \text {. }
$$

Corollary 13. For any pseudofinite difference field $(F$, Frob $) \in \mathcal{S}$, the coarse dimension $\boldsymbol{\delta}_{F}$ is definable and integer-valued for all $\mathcal{L}_{\sigma}$-definable sets. Moreover, $\boldsymbol{\delta}_{F}$ is additive in the language $\mathcal{L}_{\sigma}$.

Proof. By Theorem 9, for any $\mathcal{L}_{\sigma}$-formula $\psi(x, y)$ with $|x|=1$, any $b \in F^{|y|}$ we have

$$
\boldsymbol{\delta}_{F}(\psi(F, b)) \in\{0,1\} .
$$

Applying Lemma 12 we get the desired result.

Remark: In general, the coarse dimension does not have the property that a definable set has dimension 0 if only if it is finite. Similarly, in a group, we don't necessarily have that a subgroup of infinite index will have smaller dimension.

Example 14. Let $(F$, Frob $)=\prod_{p \in \mathbb{P}}\left(\mathbb{F}_{p^{k_{p}}}, \operatorname{Frob}_{p}\right) / \mathcal{U} \in \mathcal{S}$. Define a function $f: F^{\times} \rightarrow$ $F^{\times}$as

$$
f(x):=x^{-1} \cdot \operatorname{Frob}(x) .
$$

It is easy to see that $f$ is a group homomorphism. Therefore, the image $T:=f\left(F^{\times}\right)$is a definable subgroup of $F^{\times}$. There is a corresponding $f_{p}:\left(\mathbb{F}_{p^{k_{p}}}\right)^{\times} \rightarrow\left(\mathbb{F}_{p^{k_{p}}}\right)^{\times}$and $T_{p}:=$ $f_{p}\left(\left(\mathbb{F}_{p^{k_{p}}}\right)^{\times}\right)$for any $p \in \mathbb{P}$. Since the kernel of $f_{p}$ is $\left(\mathbb{F}_{p}\right)^{\times}$, we get $\left[\left(\mathbb{F}_{p^{k_{p}}}\right)^{\times}: T_{p}\right]=p-1$. Hence, $T$ has infinite index in $F^{\times}$, though $\boldsymbol{\delta}_{F}(T)=\boldsymbol{\delta}_{F}\left(F^{\times}\right)$.

\section{Coarse dimension and transformal transcendence degree}

In the following, we will try to understand whether there are some algebraic properties of difference fields that are intrinsic to the coarse dimension $\boldsymbol{\delta}_{F}$.

Let us start with an observation. Given $(F$, Frob $)=\left(\mathbb{F}_{p^{k_{p}}}\right.$, Frob $\left._{p}\right) / \mathcal{U} \in \mathcal{S}$. Let

$$
(\tilde{F}, \text { Frob }):=\prod_{p \in \mathbb{P}}\left(\tilde{\mathbb{F}}_{p}, \text { Frob }_{p}\right) / \mathcal{U}
$$

then $(\tilde{F}$, Frob) is a model of ACFA, which contains $(F$, Frob) as a substructure.

In ACFA, there is a notion of dimension which is also integer-valued, and it is induced by SU-rank.

Let $\mathbf{k}$ be a saturated model of ACFA.

Definition 15. Let $a$ be a finite tuple in $\mathbf{k}$ and $A \subseteq \mathbf{k}$. Then $S U(a / A)=\omega \cdot k+n$ for some $0 \leq k \leq|a|$. Define the rank-dimension $\operatorname{dim}_{r k}$ of $\operatorname{tp}(a / A)$ as $\operatorname{dim}_{r k}(a / A):=k$. 
Remark: $\operatorname{dim}_{r k}(a / A)$ coincides with the transformal transcendence degree of $a$ over $A_{\sigma}$ (the difference field generated by $A$ ).

Now we have two integer-valued additive dimensions on definable sets: $\operatorname{dim}_{r k}$ and the coarse dimension $\boldsymbol{\delta}_{F}$. Note further that $\operatorname{dim}_{r k}(F)=\boldsymbol{\delta}_{F}(F)=1$ and $\operatorname{dim}_{r k}(\operatorname{Fix}(F))=$ $\boldsymbol{\delta}_{F}(\operatorname{Fix}(F))=0$. It is natural to ask whether they coincide on all definable sets.

One of the inequalities is obvious.

Lemma 16. Let $(F, F r o b) \in \mathcal{S}$. For any tuple $a \in F$ and subset $A \subseteq F$ we have $\boldsymbol{\delta}_{F}(a / A) \leq \operatorname{dim}_{r k}(a / A)$.

Proof. Note that by the additivity of both $\operatorname{dim}_{r k}$ and $\delta_{F}$, we only need to prove the inequality when $a$ is a single element. We may assume that $A=A_{\sigma}$. By [4], we know that $S U(a / A)=\omega$ if and only if $a$ is transformally transcendental over $A$ if and only if $\operatorname{deg}_{\sigma}(a / A)=\infty$. Therefore, we need to show that if $\operatorname{deg}_{\sigma}(a / A)<\infty$ then $\boldsymbol{\delta}_{F}(a / A)=0$.

Suppose $\operatorname{deg}_{\sigma}(a / A)<\infty$. Then there is some $m$ and a non-trivial polynomial $f\left(x ; y_{1}, \ldots, y_{m}\right)$ with parameters in $A$, such that $f\left(\sigma^{m}(a) ; \sigma^{m-1}(a), \ldots, a\right)=0$. Take any prime $p \in \mathbb{P}$ and let $g_{p}(x):=f\left(x^{p^{m}} ; x^{p^{m-1}}, \ldots, x\right)$. Then $\left|g_{p}\left(\mathbb{F}_{p^{k_{p}}}\right)=0\right| \leq p^{C \cdot m}$ for some constant $C$ depending on $f$. Let $\varphi(x):=f\left(\sigma^{m}(x) ; \sigma^{m-1}(x), \ldots, x\right)=0$. Then $\varphi(x)$ defines exactly the set $g_{p}\left(\mathbb{F}_{p^{k_{p}}}\right)=0$ in $\left(\mathbb{F}_{p^{k_{p}}}\right.$, Frob $\left._{p}\right)$. Therefore, $\boldsymbol{\delta}_{F}(\varphi(F))=0$. As $a \in \varphi(F)$, we get $\boldsymbol{\delta}_{F}(a / A)=0$.

We conjecture that in general the two dimensions coinside. But at the moment, we can only prove the case for existential formulas. To prove this, we will use the estimation of the number of solutions of formulas in ACFA, which is given in [11] based on Hrushovski's twisted Lang-Weil estimate.

Definition 17. Let $\varphi(x)$ be a difference formula with parameters $A$. We define

$$
\operatorname{deg}_{\sigma}(\varphi(x)):=\max \left\{\operatorname{deg}_{\sigma}\left(a / A_{\sigma}\right): \varphi(a) \text { holds }\right\}
$$

Remark: Given a formula $\varphi(x, y)$, seen as a family of definable sets parametrised by the variable $y$, by $[4$, Section 7$]$, the set $\left\{y: \operatorname{deg}_{\sigma}(\varphi(x, y))=d\right\}$ is definable.

Fact 18. [11, Theorem 1.1] and [12, Theorem 2.1.1] Let $K_{q}:=\left(\tilde{\mathbb{F}}_{p}, \Phi_{q}: x \mapsto x^{q}\right)$ where $q$ is a power of the prime number $p$. Let $\varphi(x, y)$ be a formula in the language of difference rings, with $x=\left(x_{1}, \ldots, x_{n}\right)$ and $y=\left(y_{1}, \ldots, y_{m}\right)$. Then there is a positive constant $C$ and a finite set $D$ of pairs $(d, \mu)$ with $D \subseteq \mathbb{Z}$ and $\mu \in \mathbb{Q}^{+}$, such that in each field $K_{q}$ and each $y_{0} \in K_{q}^{m}$, one of the following happens:

1. There are some $(d, \mu) \in D$ such that $\operatorname{deg}_{\sigma}\left(\varphi\left(x, y_{0}\right)\right)=d$, and we have the estimate

$$
|| \varphi\left(K_{q}^{n}, y_{0}\right)\left|-\mu q^{d}\right| \leq C q^{d-\frac{1}{2}} .
$$

2. $\operatorname{deg}_{\sigma}\left(\varphi\left(x, y_{0}\right)\right)=\infty$ and $\left|\varphi\left(K_{q}^{n}, y_{0}\right)\right|=\infty$.

Lemma 19. Let a be a tuple in $F$ and $A \subseteq F$. Suppose the coarse dimension $\boldsymbol{\delta}_{F}(a / A)$ is witnessed by an existential formula, that is, there is some formula $\exists y \psi(x, y)$ with $\psi(x, y)$ quantifier-free (possibly with parameters), such that $\boldsymbol{\delta}_{F}(\exists y \psi(F|x|, y))=\boldsymbol{\delta}_{F}(a / A)$ and $\exists y \psi(x, y) \in t p(a / A)$. Then $\boldsymbol{\delta}_{F}(a / A)=\operatorname{dim}_{r k}(a / A)$. 
Proof. We can write $a=a_{1} a_{2}$ where $\boldsymbol{\delta}_{F}(a / A)=\boldsymbol{\delta}_{F}\left(a_{1} / A\right)=\left|a_{1}\right|$. Suppose that $(F$, Frob $)=\varphi\left(a_{1}, a_{2}, a^{\prime}\right)$ with $a^{\prime} \subseteq A$ witnesses the coarse dimension of $a$ over $A$ and that $\varphi\left(x_{1}, x_{2}, y\right):=\exists z \psi\left(x_{1}, x_{2}, y, z\right)$, where $\psi\left(x_{1}, x_{2}, y, z\right)$ is quantifier-free. We claim that $\boldsymbol{\delta}_{F}\left(\varphi\left(a_{1}, F^{\left|x_{2}\right|}, a^{\prime}\right)\right)=0$. If not, then let $b \in F^{\left|x_{2}\right|}$ such that $\left(F\right.$, Frob) $\models \varphi\left(a_{1}, b, a^{\prime}\right)$ and $\boldsymbol{\delta}_{F}\left(b / a^{\prime}, a_{1}\right)=\boldsymbol{\delta}_{F}\left(\varphi\left(a_{1}, F^{\left|x_{2}\right|}, a^{\prime}\right)\right)>0$. Then

$$
\boldsymbol{\delta}_{F}\left(a_{1}, b / a^{\prime}\right)=\boldsymbol{\delta}_{F}\left(a_{1} / a^{\prime}\right)+\boldsymbol{\delta}_{F}\left(b / a^{\prime}, a_{1}\right)>\boldsymbol{\delta}_{F}\left(a_{1} / a^{\prime}\right)=\boldsymbol{\delta}_{F}\left(a / a^{\prime}\right) .
$$

Since $\left(F\right.$, Frob) $\mid=\varphi\left(a_{1}, b, a^{\prime}\right)$, we get $\boldsymbol{\delta}_{F}\left(\varphi\left(F^{\left|x_{1} x_{2}\right|}, a^{\prime}\right)\right) \geq \boldsymbol{\delta}_{F}\left(a_{1}, b / a^{\prime}\right)>\boldsymbol{\delta}_{F}\left(a / a^{\prime}\right)$. This contradicts our assumption that $\varphi\left(x_{1}, x_{2}, a^{\prime}\right)$ witnesses $\boldsymbol{\delta}_{F}\left(a / a^{\prime}\right)$.

By Lemma 16, we have

$$
\left|a_{1}\right|=\boldsymbol{\delta}_{F}\left(a_{1} / a^{\prime}\right) \leq \operatorname{dim}_{r k}\left(a_{1} / a^{\prime}\right) \leq\left|a_{1}\right| .
$$

Therefore, $\operatorname{dim}_{r k}\left(a_{1} / a^{\prime}\right)=\boldsymbol{\delta}_{F}\left(a_{1} / a^{\prime}\right)=\boldsymbol{\delta}_{F}\left(a / a^{\prime}\right)$. To show that $\operatorname{dim}_{r k}\left(a / a^{\prime}\right)=\boldsymbol{\delta}_{F}\left(a / a^{\prime}\right)$, we only need to show that $\operatorname{dim}_{r k}\left(a_{2} / a^{\prime}, a_{1}\right)=0$. Therefore, we need to prove the following claim:

Let $\varphi(x, b)$ (with $b \in F$ a tuple) be an existential formula in the language of diffence rings such that $\boldsymbol{\delta}_{F}(\varphi(x, b))=0$. Then for any tuple $a \in F$ with $(F$, Frob $) \models \varphi(a, b)$, we have $\operatorname{deg}_{\sigma}(a / b)<\infty$.

Suppose $a=\left(a_{p}\right)_{p \in \mathbb{P}} / \mathcal{U}$ and $b=\left(b_{p}\right)_{p \in \mathbb{P}} / \mathcal{U}$. Let $\varphi_{p}(x, y)$ be defined as in Definition 7. As $\boldsymbol{\delta}_{F}(a / A)=0$, by our construction, there is some $V$ in the ultrafilter $\mathcal{U}$ which has the following property: for all $p \in V$, there is a constant $C_{p}$ such that for all $k$ with $b_{p} \in \mathbb{F}_{p^{k}}$, we have $\left|\varphi_{p}\left(\mathbb{F}_{p^{k}}, b_{p}\right)\right| \leq C_{p}$.

We claim that $\left|\varphi_{p}\left(\tilde{\mathbb{F}}_{p}, b_{p}\right)\right| \leq C_{p}$. Suppose not, then take $\left\{a_{0}, a_{1}, \ldots, a_{\left\lceil C_{p}\right\rceil}\right\} \subseteq$ $\varphi_{p}\left(\left(\tilde{\mathbb{F}}_{p}\right)^{|x|}, b_{p}\right)$. As $\varphi(x, y)$ is existential, so is $\varphi_{p}(x, y)$. We may suppose $\varphi_{p}(x, y)=$ $\exists z \psi_{p}(x, y, z)$. For each $a_{i}$, pick some $e_{i} \in\left(\tilde{\mathbb{F}}_{p}\right)^{|z|}$ such that $\tilde{\mathbb{F}}_{p} \models \psi_{p}\left(a_{i}, b_{p}, e_{i}\right)$. Let $\mathbb{F}_{p^{k}}$ be a large finite field contains all the points $\left\{a_{0}, \ldots, a_{\left\lceil C_{p}\right\rceil}, e_{0}, \ldots, e_{\left\lceil C_{p}\right\rceil}, b_{p}\right\}$, then we have

$$
\operatorname{card}\left(\varphi_{p}\left(\mathbb{F}_{p^{k}}, b_{p}\right)\right) \geq\left\lceil C_{p}\right\rceil+1>C_{p}
$$

contradiction.

Let $K_{p}:=\left(\tilde{\mathbb{F}}_{p}, \Phi_{p}: x \mapsto x^{p}\right)$. Note that $\varphi_{p}\left(\tilde{\mathbb{F}}_{p}, b_{p}\right)$ is exactly the set $\varphi\left(K_{p}, b_{p}\right)$. Then by Fact 18 and that $\left|\varphi\left(K_{p}, b_{p}\right)\right|=\left|\varphi_{p}\left(\tilde{\mathbb{F}}_{p}, b_{p}\right)\right|<\infty$ for each $b_{p}$, we get a finite set $D$ of pairs $(d, \mu) \in \mathbb{N} \times \mathbb{Q}^{+}$such that for any $b_{p}$, there is some $(d, \mu) \in D$ and the following holds:

$$
|| \varphi\left(K_{p}, b_{p}\right)\left|-\mu p^{d}\right| \leq C p^{d-\frac{1}{2}} .
$$

Therefore, there is some $J \in \mathcal{U}$ and one particular pair $(d, \mu) \in D$ such that for any $p \in$ $J$, we have $\left|\operatorname{card}\left(\varphi\left(K_{p}, b_{p}\right)\right)-\mu p^{d}\right| \leq C p^{d-\frac{1}{2}}$. By Fact 18 we know that $\operatorname{deg}_{\sigma}\left(\varphi\left(x, b_{p}\right)\right)=d$ for any $b_{p} \in J$. By the subsequent remark, we know there is some formula $\varphi_{d}(y)$, such that $\varphi_{d}(y)$ holds if and only if $\operatorname{deg}_{\sigma}(\varphi(x, y))=d$ in a difference field. Therefore, $\varphi_{d}\left(b_{p}\right)$ holds in each $K_{p}$ with $p \in J$, hence $\varphi_{d}(b)$ holds in $(\tilde{F}$, Frob). As $\varphi(x, y)$ is an existential formula, $(F$, Frob $) \models \varphi(a, b)$ implies $(\tilde{F}$, Frob $) \models \varphi(a, b)$. We conclude that

$$
\operatorname{deg}_{\sigma}(a / b) \leq \operatorname{deg}_{\sigma}(\varphi(x, b))=d .
$$

The previous Lemma says essentially that if a set is definable by a pure existential formula, then all the elements of maximal coarse dimension, the "generic elements", can be controlled by their quantifier-free type. It would be nice to also have some control over those "non-generic" elements. It turned out that this can be done. 
Lemma 20. Let $\varphi(x):=\exists y \psi(x, y)$ be an $\mathcal{L}_{\sigma}$-formula such that $\psi(x, y)$ is quantifier-free with parameters in the finite set $A \subseteq F$. Then for any $a \in F^{|x|}$ with $(F$, Frob $) \models \varphi(a)$, we have $\operatorname{dim}_{r k}(a / A) \leq \boldsymbol{\delta}_{F}\left(\varphi\left(F^{|x|}\right)\right)$.

Proof. Let $n$ be the length of the tuple $x$ in $\varphi(x)$. Suppose $a \in F^{n}$ and $(F$, Frob $) \models \varphi(a)$. Denote the set of complete quantifier-free $n$-types over $A$ as $S_{n}^{q f}(A)$. Hence, there is some $p \in S_{n}^{q f}(A)$ such that $(F$, Frob $) \models \varphi(a) \wedge p(a)$. Clearly,

$$
t:=\boldsymbol{\delta}_{F}(\{\varphi\} \cup p):=\min \left\{\boldsymbol{\delta}_{F}\left(\psi\left(F^{n}\right) \wedge \varphi\left(F^{n}\right)\right): \psi \in p\right\} \leq \boldsymbol{\delta}_{F}\left(\varphi\left(F^{n}\right)\right) .
$$

By the extension property (every partial type extents to a complete type of the same coarse dimension) and $\omega$-saturation of $\left(F\right.$, Frob), there is some $a^{\prime} \in F^{n}$ such that $\boldsymbol{\delta}_{F}\left(a^{\prime} / A\right)=\boldsymbol{\delta}_{F}(\{\varphi\} \cup p)=t$. Hence, $\boldsymbol{\delta}_{F}\left(a^{\prime} / A\right)=\boldsymbol{\delta}_{F}\left(\varphi\left(F^{n}\right) \wedge \psi\left(F^{n}\right)\right)$ for some $\psi(x) \in p$. As $p$ is quantifier-free, by Lemma 19 , we have $\operatorname{dim}_{r k}\left(a^{\prime} / A\right)=t$. Since $a$ and $a^{\prime}$ have the same quantifier-free type $p$ over $A$, we must have

$$
\operatorname{dim}_{r k}(a / A)=\operatorname{dim}_{r k}\left(a^{\prime} / A\right)=t \leq \boldsymbol{\delta}_{F}\left(\varphi\left(F^{n}\right)\right) .
$$

This partial connection between $\operatorname{dim}_{r k}$ and $\boldsymbol{\delta}_{F}$ already can help us to establish more properties of $(F$, Frob). The strategy is the following: we start with a definable object in $\left(F\right.$, Frob). If we have the control over $\operatorname{dim}_{r k}$ of elements in it, then we work in $(\tilde{F}$, Frob). As it is a model of ACFA, we can use all the model theoretic tools there. In the end, we transfer the results in $(\tilde{F}$, Frob) back to those in $(F$, Frob).

Fact 21. Let $(k, \sigma)$ be a model of ACFA. Let $G$ be a definable subgroup of some algebraic group $H(k)$. Let acl $\sigma$ denote the algebraic closure in ACFA. Suppose $G$ is definable over $E=\operatorname{acl}_{\sigma}(E)$. Then $G$ is contained in a group $\tilde{G}$ which is quantifier-free definable over $E$ and has the same SU-rank as $G$.

Remark: This statement can be found in [3, Section 6.5].

Notation: For a difference formula $\varphi(x)$ with parameters in $A \subseteq(\tilde{F}$, Frob $)$. Let

$$
d=\max \left\{n \leq|x|: S U(a / A)=\omega \cdot n+m \text {, for some } a \in \varphi\left((\tilde{F})^{|x|}\right)\right\} .
$$

We define $\operatorname{dim}_{r k}(\varphi(x)):=d$.

Lemma 22. Let $(F$, Frob $) \in \mathcal{S}$. Given $a \in F^{n}$ and $A \subseteq F$. Suppose $\operatorname{dim}_{r k}(a / A)=k$. Then there is a finite set $\left\{P_{1}(x), \ldots, P_{m}(x)\right\}$ of difference polynomials with parameters in $A$ such that $(F$, Frob $) \models \bigwedge_{i \leq m} P_{i}(a)=0$ and $\operatorname{dim}_{r k}\left(\bigwedge_{i \leq m} P_{i}(x)=0\right)=k$.

Proof. We may write $a$ into two parts $a_{1}$ and $a_{2}$ where $\operatorname{dim}_{r k}\left(a_{1} / A\right)=\left|a_{1}\right|=k$, and $\operatorname{dim}_{r k}\left(a_{2} / A a_{1}\right)=0$. Let $\left(A a_{1}\right)_{\sigma}$ be the difference field generated by $A \cup\left\{a_{1}\right\}$. Suppose $a_{2}:=a_{2}^{1} \cdots a_{2}^{m}$ with each $\left|a_{2}^{i}\right|=1$. Since $\operatorname{dim}_{r k}\left(a_{2}^{i} / A a_{1}\right)=0$ for each $i \leq m$, we get $\operatorname{deg}_{\sigma}\left(a_{2}^{i} /\left(A a_{1}\right)_{\sigma}\right)<\infty$. Therefore, there is a difference polynomial $P_{i}\left(y_{i}, b_{i}\right)$ with $b_{i} \subseteq$ $\left(A a_{1}\right)_{\sigma}$ such that $a_{2}^{i}$ vanishes on it. Write $b_{i}=f_{i}\left(a_{1}\right)$ where $f_{i}$ is a difference polynomial with parameters in $A$. We should rearrange the order of variables such that $x_{0}, \ldots, x_{|a|-1}$ corresponds to the order of $a$. Suppose $a_{1}=a^{l_{1}} \cdots a^{l_{\left|a_{1}\right|}}$ and $a_{2}=a^{t_{1}} \cdots a^{t_{\left|a_{2}\right|} \mid}$ where $a^{j}$ is the $j^{t h}$ digit of $a$. Now it is easy to see that $a$ satisfies the formula

$$
\bigwedge_{i \leq m} P_{i}\left(x_{t_{i}}, f_{i}\left(x_{l_{1}}, \ldots, x_{l_{\left|a_{1}\right|}}\right)\right)=0
$$

and $\operatorname{dim}_{r k}\left(\bigwedge_{i \leq m} P_{i}\left(x_{t_{i}}, f_{i}\left(x_{l_{1}}, \ldots, x_{l_{\left|a_{1}\right|}}\right)\right)=0\right)=k$. 
Corollary 23. Let $(F$, Frob) $\in \mathcal{S}$. Suppose $G$ is a definable (possibly with parameters in $F$ ) subgroup of some algebraic group $H(F) \subseteq F^{t}$. If $G$ is defined by some existential formula, then there is a quantifier-free definable group $\bar{G} \geq G$ (defined with parameters in $F)$, such that $\boldsymbol{\delta}_{F}(\bar{G})=\boldsymbol{\delta}_{F}(G)$.

Proof. Suppose $G$ is defined over the finite set $A \subseteq F$ with the formula $\varphi_{G}$. Let $k:=\boldsymbol{\delta}_{F}(G)$.

Let $\Pi_{A}$ be the set of difference polynomials in $t$-variables with coefficients in $A$.

By Lemma 22, for any element $a \in G$, there are some $\left\{P_{a, i}(x): 1 \leq i \leq m_{a}\right\} \subset \Pi_{A}$ such that $(F$, Frob $) \models \bigwedge_{i \leq m_{a}} P_{a, i}(a)=0$ and $\operatorname{dim}_{r k}\left(\bigwedge_{i \leq m_{a}} P_{a, i}(x)=0\right)=\operatorname{dim}_{r k}(a / A)$. By Lemma 20, $\operatorname{dim}_{r k}(a / \bar{A}) \leq \boldsymbol{\delta}_{F}(G)=k$.

Therefore, $\varphi_{G}(x) \models \bigvee_{a \in G}\left(\bigwedge_{i \leq m_{a}} P_{a, i}(x)=0\right.$ ). (The right hand-side is a countable disjunction, since $P_{a, i}(x)=0$ are in $\mathcal{L}_{\sigma} \cup\{A\}$ and $A$ is a finite set.) By compactness, there is some finite set $a_{0}, \ldots, a_{l}$ such that

$$
\varphi_{G}(x) \models \bigvee_{j \leq \ell}\left(\bigwedge_{i \leq m_{a_{j}}} P_{a_{j}, i}(x)=0\right) .
$$

As $\operatorname{dim}_{r k}\left(\bigwedge_{i \leq m_{a_{j}}} P_{a_{j}, i}(x)=0\right) \leq k$ for each $j \leq \ell$, we get

$$
\operatorname{dim}_{r k}\left(\bigvee_{j \leq \ell}\left(\bigwedge_{i \leq m_{a_{j}}} P_{a_{j}, i}(x)=0\right)\right) \leq k
$$

Write the formula $\bigvee_{j \leq \ell}\left(\bigwedge_{i \leq m_{a_{j}}} P_{a_{j}, i}(x)=0\right)$ into the conjunctive normal form

$$
\bigwedge_{u \leq N} \bigvee_{v \leq M_{u}}\left(P_{u, v}(x)=0\right)
$$

for some natural numbers $N, M_{u}$, and each $P_{u, v}(x) \in\left\{P_{a_{j}, i}(x): j \leq \ell, i \leq m_{a_{j}}\right\}$. Hence, for each $u \leq N$, we have $\varphi_{G}(x) \models\left(\prod_{v \leq M_{u}} P_{u, v}(x)\right)=0$.

Let $G_{\tilde{F}}$ be closure of $G$ under the $\sigma$-Zariski topology in $(\tilde{F}$, Frob), that is, if we define $I_{\tilde{F}}(G)=\left\{p \in \tilde{F}[x]_{\sigma}: p(g)=0\right.$ for all $\left.g \in G\right\}$, then

$$
G_{\tilde{F}}:=\left\{h \in H(\tilde{F}): p(h)=0 \text { for all } p \in I_{\tilde{F}}(G)\right\} .
$$

As prime $\sigma$-ideals are finitely generated, $G_{\tilde{F}}$ is quantifier-free definable. Note that $\prod_{v \leq M_{u}} P_{u, v}(x) \in I_{\tilde{F}}(G)$ for each $u \leq N$. Since

$$
\operatorname{dim}_{r k}\left(\bigwedge_{u \leq N}\left(\prod_{v \leq M_{u}} P_{u, v}(x)\right)=0\right)=\operatorname{dim}_{r k}\left(\bigvee_{j \leq \ell}\left(\bigwedge_{i \leq m_{a_{j}}} P_{a_{j}, i}(x)=0\right)\right) \leq k,
$$

we get $\operatorname{dim}_{r k}\left(G_{\tilde{F}}\right) \leq k$.

Take an automorphism $\delta$ of $\left(\tilde{F}\right.$, Frob) fixing $F$. Then $G=\delta(G) \subseteq \delta\left(G_{\tilde{F}}\right)$. As $\delta\left(G_{\tilde{F}}\right)$ is also closed under the $\sigma$-Zariski topology in $\left(\tilde{F}\right.$, Frob), we get $G_{\tilde{F}} \subseteq \delta\left(G_{\tilde{F}}\right)$ which implies $G_{\tilde{F}}=\delta\left(G_{\tilde{F}}\right)$. Therefore, $G_{\tilde{F}}$ is invariant under automorphisms fixing $F$, hence it is definable over $F$. Let $E=\operatorname{acl}_{\sigma}(F)=F^{a l g}$, then by Fact 21, there is $G_{E}$ which contains $G_{\tilde{F}}$, has the same $S U$-rank as $G_{E}$ and is quantifier-free definable over 
$E$. In fact, $G_{E}$ is the smallest closed set containing $G_{\tilde{F}}$ in the $\sigma$-Zariski topology in $\left(F^{\text {alg }}\right.$, Frob $\left\lceil_{F^{a l g}}\right)$.

Suppose $G_{E}$ is defined by

$$
\bigwedge_{0 \leq j \leq \ell} P_{j}\left(x, \sigma(x), \ldots, \sigma^{m}(x), a_{j}\right)=0
$$

where $P_{j}$ are polynomials in the language of rings and $a_{j} \subseteq F^{a l g}$. For any $0 \leq j \leq \ell$, let $\left\{a_{j}^{0}, \ldots, a_{j}^{N_{j}}\right\} \subseteq\left(F^{a l g}\right)^{\left|a_{j}\right|}$ be the set of all field conjugates of $a_{j}$ over $F$. Note that for any $g \in G$ we have $g, \sigma(g), \ldots, \sigma^{m}(g) \subseteq F$. Hence, $P_{j}\left(g, \sigma(g), \ldots, \sigma^{m}(g), a_{j}\right)=0$ if and only if $P_{j}\left(g, \sigma(g), \ldots, \sigma^{m}(g), a_{j}^{i}\right)=0$ for any $g \in G$ and $0 \leq i \leq N_{j}$.

Let $B_{j}$ be the set in $H(\tilde{F})$ vanishing on $\left\{P_{j}\left(x, \sigma(x), \ldots, \sigma^{m}(x), a_{j}^{i}\right): 0 \leq i \leq N_{j}\right\}$. Then from the above argument, we know $B_{j} \supseteq G$. As $B_{j}$ is closed under the $\sigma$-Zariski topology in $\left(\tilde{F}\right.$, Frob), we get $B_{j} \supseteq G_{\tilde{F}}$. Similarly, by $B_{j}$ being closed under the $\sigma$ Zariski topology in $\left(F^{a l g}\right.$, Frob $\left.\uparrow_{F a l g}\right)$, we get $B_{j} \supseteq G_{E}$.

Now consider the following formula

$$
\bigwedge_{0 \leq j \leq \ell} \bigwedge_{0 \leq i \leq N_{j}} P_{j}\left(x, \ldots, \sigma^{m}(x), a_{j}^{i}\right)=0
$$

It defines $\bigcap_{j \leq \ell} B_{j}$. By the argument above, we know that $\bigcap_{j \leq \ell} B_{j} \supseteq G_{E}$. Clearly, we also have $\bigcap_{j \leq \ell} B_{j} \subseteq G_{E}$. Hence, the formula above also defines $G_{E}$ in $H(\tilde{F})$. Now we show that $G_{E}$ can be made quantifier-free definable over $F$.

Fix $0 \leq j \leq \ell$, consider the formula

$$
\bigwedge_{0 \leq i \leq N_{j}} P_{j}\left(x, x_{1}, \ldots, x_{m}, a_{j}^{i}\right)=0
$$

where $x_{1}, \ldots, x_{m}$ are distinct tuples of variables all have the same length as $x$. For $1 \leq k \leq N_{l}+1$, let $e_{k}\left(t_{0}, \ldots, t_{N_{j}}\right)$ be the $k$-elementary symmetric polynomials in $N_{j}+1$-variables, i.e.,

$$
e_{k}\left(t_{0}, \ldots, t_{N_{j}}\right):=\sum_{0 \leq i_{1}<\cdots<i_{k} \leq N_{j}} t_{i_{1}} \cdots t_{i_{k}}
$$

Then we have $\bigwedge_{0 \leq i \leq N_{j}} P_{j}\left(x, x_{1}, \ldots, x_{m}, a_{j}^{i}\right)=0$ if and only if

$$
\bigwedge_{1 \leq k \leq N_{j}+1} e_{k}\left(P_{j}\left(x, x_{1}, \ldots, x_{m}, a_{j}^{0}\right), \ldots, P_{j}\left(x, x_{1}, \ldots, x_{m}, a_{j}^{N_{j}}\right)=0 .\right.
$$

For each $1 \leq k \leq N_{j}+1$, as $\left\{a_{j}^{i}: 0 \leq j \leq N_{j}\right\}$ is the set of all field conjugates of $a_{j}$ in $F^{a l g}$ over $F$ and that $e_{k}$ is symmetric, we get

$$
Q_{j}^{k}\left(x, \ldots, x_{m}, b_{j}^{k}\right):=e_{k}\left(P_{j}\left(x, x_{1}, \ldots, x_{m}, a_{j}^{0}\right), \ldots, P_{j}\left(x, x_{1}, \ldots, x_{m}, a_{j}^{N_{j}}\right)\right.
$$

is invariant under field automorphisms $\operatorname{Gal}\left(F^{a l g} / F\right)$. Therefore, $b_{j}^{k} \subseteq F$ (since $F$ is perfect). 
Let $\varphi_{H}(x)$ be the quantifier-free formula with parameters in $A$ that defines the algebraic group $H$. Now consider

$$
\psi(x):=\varphi_{H}(x) \wedge\left(\bigwedge_{0 \leq j \leq \ell} \bigwedge_{1 \leq k \leq N_{j}+1} Q_{j}^{k}\left(x, \sigma(x), \ldots, \sigma^{m}(x), b_{j}^{k}\right)\right) .
$$

It is easy to see that $\psi(x)$ defines $G_{E}$ in $(\tilde{F}$, Frob). As $\psi(x)$ is quantifier-free and defined over $F$, we can consider $\bar{G}:=\left\{g \in F^{t}:(F\right.$, Frob $\left.) \models \psi(g)\right\}$. As $H(F)$ is an algebraic group and $F$ is definably closed in $\tilde{F}$ in the language of rings, $\bar{G}$ is a quantifier-free definable group in $\left(F\right.$, Frob) and contains $G$. Note that $\operatorname{dim}_{r k}\left(G_{E}\right)=\operatorname{dim}_{r k}\left(G_{\tilde{F}}\right) \leq k$. Hence, $\boldsymbol{\delta}_{F}(\bar{G}) \leq \operatorname{dim}_{r k}(\psi(x))=\operatorname{dim}_{r k}\left(G_{E}\right) \leq k$. On the other hand, since $\bar{G} \supseteq G$ and $\boldsymbol{\delta}_{F}(G)=k$, we get $\boldsymbol{\delta}_{F}(\bar{G}) \geq k$. Therefore, $\boldsymbol{\delta}_{F}(\bar{G})=\boldsymbol{\delta}_{F}(G)=k$.

\section{Non-tameness}

This section investigates whether this family of difference fields is tame in terms of Shelah's classification. It turns out that the answer is negative.

In the following, we will prove that if a structure expands a pseudofinite field with a "logarithmically small" definable subset, then the theory has TP2 and the strict order property and is not decidable. This result is known among experts. As we could not find a proof in the literature, we include it here for completeness.

The proof is based on the result that the theory of pseudofinite fields has the independence property in [7]. The strategy is to modify Duret's proof to show that when a pseudofinite set is very small compared to the size of the field, then every pseudofinite subset of it can also be coded uniformly.

Fact 24. ([7, Proposition 4.3]) Let $k$ is a field and $p$ a prime different from $\operatorname{char}(k)$ such that $k$ contains a $p^{\text {th }}$-root of unity. Let $\tilde{k}$ be the algebraic closure of $k$. Suppose $f_{i} \in k\left[Y_{1}, \cdots, Y_{m}\right]$ and $F_{i}=X^{p}-f_{i} \in k\left[Y_{1}, \cdots, Y_{m}, X\right]$ for $1 \leq i \leq n$. If there exist $g_{i}, h_{i} \in \tilde{k}\left[Y_{1}, \cdots, Y_{m}\right]$ and $q_{i} \in \mathbb{N}$ such that:

- for all $i, f_{i}=g_{i}^{q_{i}} h_{i}$;

- for all $i, g_{i}$ is prime in $\tilde{k}\left[Y_{1}, \cdots, Y_{m}\right]$

- for all $i \neq j, g_{i} \neq g_{j}$

- for all $i$ and $j, g_{i}$ does not divide $h_{j}$

- for all $i, p$ does not divide $q_{i}$.

Then the ideal $J$ in $k\left[Y_{1}, \cdots, Y_{m}, X_{1}, \cdots, X_{n}\right]$ generated by $\left\{F_{i}\left(X_{i}\right): 1 \leq i \leq n\right\}$ is absolutely prime, and does not contain any non-zero element in $k\left[Y_{1}, \cdots, Y_{m}\right]$.

Fact 25. ([2, Theorem 7.1]) Let $V \subseteq\left(\tilde{\mathbb{F}}_{q}\right)^{n}$ be an absolutely irreducible $\mathbb{F}_{q \text {-variety of }}$ dimension $r>0$ and degree $\delta$. If $q>2(r+1) \delta^{2}$, then the following estimate holds:

$$
||\left(V \cap\left(\mathbb{F}_{q}\right)^{n}\right)\left|-q^{r}\right| \leq(\delta-1)(\delta-2) q^{r-\frac{1}{2}}+5 \delta^{\frac{13}{3}} q^{r-1} .
$$


Theorem 26. Let $F=\prod_{i \in I} \mathbb{F}_{p_{i} n_{i}} / \mathcal{U}$ be a pseudofinite field and $A=\prod_{i \in I} A_{i} / \mathcal{U}$ a infinite pseudofinite subset of $F$. Suppose there is a constant $C$ such that $\left|A_{i}\right| \leq C n_{i}$ for any $i \in I$. Then all pseudofinite subsets of $A$ are uniformly definable.

Proof. Consider the finite algebraic extension $F^{\prime}$ of $F$ of degree $14 C$. As $F$ is pseudofinite, there is only one such extension and is definable. To see the definability, suppose $F^{\prime}=F(\alpha)$. Let $f$ be the minimal polynomial of $\alpha$ over $F$. Then we can define $F^{\prime}$ as the $14 C$-dimensional vector space over $F$ with multiplication defined according to the minimal polynomial $f$.

We distinguish two cases according to $p_{i}$.

Suppose $p_{i} \neq 2$. Since $x^{p_{i}^{14 C n_{i}}-1}=1$ for all $x \in \mathbb{F}_{p_{i}^{14 C n_{i}}}$, the square root of unity exists in $\mathbb{F}_{p_{i}}^{14 C n_{i}}$. As the multiplicative group of $\mathbb{F}_{p_{i}}^{14 C n_{i}}$ is cyclic, take $\delta_{i} \in \mathbb{F}_{p_{i}^{14 n_{i}}}$ a

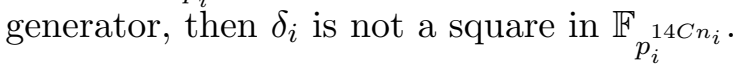

Claim 27. Let $\varphi(y, u)$ be the formula:

$$
\exists x\left(x^{2}=y+u\right) .
$$

Then for all $i \in I$ with $p_{i} \neq 2$ and for all $C_{i} \subseteq A_{i}$, there is $y_{i} \in F_{p_{i}^{14 C n_{i}}}$ such that

$$
C_{i}=\varphi\left(y_{i}, \mathbb{F}_{p_{i}^{14 C n_{i}}}\right) \cap A_{i} .
$$

Proof. Given $i \in I$ with $p_{i} \neq 2$ and $C_{i} \subseteq A_{i}$. Let $J$ be the ideal in $\mathbb{F}_{p_{i}^{14 C n_{i}}}\left[X_{1}, \cdots, X_{t_{i}}, Y\right]$ generated by

$$
\left\{X_{j}^{2}-\left(Y+c_{j}\right): c_{j} \in C_{i}\right\} \cup\left\{X_{j}^{2}-\delta_{i}\left(Y+d_{j}\right): d_{j} \in A_{i} \backslash C_{i}\right\},
$$

where $\delta_{i}$ is a generator of $\mathbb{F}_{p_{i}^{*}}^{14 n_{i}}$ as defined before. Let $V(J)$ be the corresponding

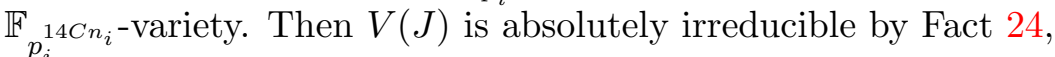

Suppose $V(J) \cap\left(\mathbb{F}_{p_{i}^{14 C n_{i}}}\right)^{t_{i}+1} \neq \emptyset$. Let $\left(x_{1}, \cdots, x_{t_{i}}, y_{i}\right)$ be a solution. Then clearly $C_{i} \subseteq \varphi\left(y_{i}, \mathbb{F}_{p_{i}}^{14 C n_{i}}\right)$. On the other hand, if there is $d \in A_{i} \backslash C_{i}$, such that $\varphi\left(y_{i}, d\right)$. Then there are $x_{j}, x \in \mathbb{F}_{p_{i}^{14 C n_{i}}}$ such that:

$$
\begin{array}{r}
x_{j}^{2}=\delta_{i}\left(y_{i}+d\right) ; \\
x^{2}=y_{i}+d ; \\
y_{i}-d \neq 0,
\end{array}
$$

where the last inequality follows from Fact 24 since $Y-d \notin J)$ Hence, $\delta_{i}=\left(x_{j} / x\right)^{2}$, contradicting that $\delta_{i}$ is not a square root. Therefore, $C_{i}=\varphi\left(y_{i}, \mathbb{F}_{p_{i}}^{14 C n_{i}}\right) \cap A_{i}$.

So we only need to show $V(J) \cap \mathbb{F}_{p_{i}^{14 C n_{i}}} \neq \emptyset$.

Let $\left|A_{i}\right|=t_{i} \leq C n_{i}$. We calculate the dimension and the degree of $V(J)$. It is clear that the dimension of $V(J)$ is 1 , as all $X_{j}$ are algebraic over $Y$. Let $c_{1}, \cdots, c_{t_{i}}$ be a list of elements in $A_{i}$. And for $1 \leq j \leq t_{i}$, let $V_{j}$ be the variety defined as the set of solutions of $X_{j}^{2}-\left(Y+c_{j}\right)$ if $c_{j} \in C_{i}$, and of $X_{j}^{2}-\delta_{i}\left(Y+c_{j}\right)$ if $c_{j} \notin C_{i}$. Then $V(J)=\bigcap_{1 \leq j \leq t_{i}} V_{j}$ and each $V_{j}$ has degree 2. Therefore, by the Bézout inequality, the degree of $V(J)$ is less than or equal to $2^{t_{i}}$. 
Suppose, towards a contradiction, that $V(J) \cap\left(\mathbb{F}_{p_{i}}^{14 C n_{i}}\right)^{t_{i}+1}=\emptyset$. Then by Fact 25 ,

$$
\begin{array}{r}
p_{i}^{14 C n_{i}} \\
\leq\left(2^{t_{i}}-1\right)\left(2^{t_{i}}-2\right) p_{i}^{7 C n_{i}}+5 \times 2^{\frac{13}{3} t_{i}} \\
\leq\left(2^{C n_{i}}-1\right)\left(2^{C n_{i}}-2\right) p_{i}^{7 C n_{i}}+5 \times 2^{\frac{13}{3} C n_{i}} \\
\leq 2^{2 C n_{i}} p_{i}^{7 C n_{i}}+2^{8 C n_{i}} \\
<p_{i}^{9 C n_{i}}+p_{i}^{8 C n_{i}} \\
<p_{i}^{14 C n_{i}},
\end{array}
$$

contradiction.

The case $p_{i}=2$ is similar. Since 3 divides $2^{14 C n_{i}}-1$ for each $i$, there exists $x \in \mathbb{F}_{2^{14 C n_{i}}}$ such that $x^{3}=1$. Take $\delta_{i}$ be the generator of the multiplicative group of $\mathbb{F}_{2^{14 C n_{i}}}$. Then there is no $y \in \mathbb{F}_{2^{14 C n_{i}}}$ such that $y^{3}=\delta_{i}$.

Claim 28. Let $\psi(y, u)$ be the formula:

$$
\exists x\left(x^{3}=y+u\right) .
$$

Then for all $i \in I$ and $C_{i} \subseteq A_{i}$, there is $y_{i} \in \mathbb{F}_{2^{14 C n_{i}}}$ such that $C_{i}=\psi\left(y_{i}, \mathbb{F}_{2^{14 C n_{i}}}\right) \cap A_{i}$.

Proof. Fix some $i$ and $C_{i} \subseteq A_{i}$. Let $J$ be the ideal in $\mathbb{F}_{2^{14 C n_{i}}}\left[X_{1}, \cdots, X_{t_{i}}, Y\right]$ generated by

$$
\left\{X_{j}^{3}-\left(Y+c_{j}\right): c_{j} \in C_{i}\right\} \cup\left\{X_{j}^{3}-\delta_{i}\left(Y+d_{j}\right): d_{j} \in A_{i} \backslash C_{i}\right\} .
$$

As the argument before, the variety $V(J)$ is absolutely irreducible of dimension 1 and of degree less than or equal to $3^{t_{i}}$. To prove the claim, we only need to show that $V(J) \cap\left(\mathbb{F}_{2^{14 C n_{i}}}\right)^{t_{i}+1} \neq \emptyset$. Suppose not, then by Fact 25 ,

$$
2^{14 C n_{i}} \leq\left(3^{t_{i}}-1\right)\left(3^{t_{i}}-2\right) 2^{7 C n_{i}}+5 \times 3^{\frac{13}{3} C t_{i}} \leq 3^{2 C n_{i}} 2^{7 C n_{i}}+3^{7 C n_{i}}<2^{14 C n_{i}},
$$

contradiction.

Let $A=\prod_{i \in I} A_{i} / \mathcal{U}$. Assume $A$ is defined by $\chi(x)$. Define $\phi(x, y):=\psi(y, x) \wedge \chi(x)$ if the characteristic of $F^{\prime}$ is 2 , and $\phi(x, y):=\varphi(y, x) \wedge \chi(x)$ otherwise. Let $C=$ $\prod_{i \in I} C_{i} / \mathcal{U} \subseteq A$ be any pseudofinite subset. By the previous two claims, there is $y_{C} \in F^{\prime}$ such that $C=\phi\left(F^{\prime}, y_{C}\right)$ in $F^{\prime}$. As $F^{\prime}$ is definable in $F$, let $\phi^{\prime}(\bar{x}, \bar{y})$ be the corresponding translation of $\phi(x, y)$ in $F$. Remember that we regard $\bar{x}, \bar{y} \in F^{\prime}$ as $14 C$-dimensional vector space over $F$ and $A \subseteq F$. Let $\theta(x, \bar{y}):=\phi^{\prime}(x, 0, \ldots, 0, \bar{y})$. We see that $\theta(x, \bar{y})$ codes uniformly all pseudofinite subsets of $A$.

Remark: From the proof we know that if $\operatorname{char}(F) \neq 2$ and $n_{i} \geq 14\left|A_{i}\right|$ for all large enough $i$, then we can take $\theta(x, \bar{y}):=\exists z^{2}\left(z^{2}=x+y\right) \wedge \chi(x)$ where $x, y$ are single variables and $\chi(x)$ is the formula defining $A$.

Corollary 29. Let $F=\prod_{i \in I} \mathbb{F}_{p_{i} n_{i}} / \mathcal{U}$ be a pseudofinite field and $B=\prod_{i \in I} B_{i} / \mathcal{U}$ an infinite pseudofinite subset of $F$. Suppose there is a constant $C$ such that $\left|B_{i}\right| \leq C n_{i}$ for all $i \in I$. Then $(F, B)$ interprets the structural $N=\prod_{i \in I}\left(N_{i},+, \times\right) / \mathcal{U}$, where $N_{i}=\left\{j \in \mathbb{N}: 0 \leq j \leq m_{i}\right\}$ for some $m_{i} \in \mathbb{N}$, and,$+ \times$ the addition and multiplication truncated on $N_{i}$ respectively. 
Proof. For each $i \in I$, pick $Y_{i} \subseteq B_{i}$ such that $\left|B_{i}\right|^{\frac{1}{4}} \leq\left|Y_{i}\right| \leq\left|B_{i}\right|^{\frac{1}{3}}$. Let $Y=\prod_{i \in I} Y_{i} / \mathcal{U}$. By Theorem 26, $Y$ is definable and all subsets of $Y_{i}$ are uniformly definable by some $\psi_{1}(y, u)$. For each $i \in I$, consider the set

$$
\frac{Y_{i}-Y_{i}}{Y_{i}-Y_{i}}:=\left\{\frac{y_{1}-y_{2}}{y_{3}-y_{4}}: y_{1}, y_{2}, y_{3}, y_{4} \in Y_{i}, y_{3} \neq y_{4}\right\}
$$

It has size at most $\left|Y_{i}\right|^{4}<<\left|\mathbb{F}_{p_{i}^{n_{i}}}\right|$. Take any $a \notin \frac{Y_{i}-Y_{i}}{Y_{i}-Y_{i}} \cup\{0\}$. Then the set $T_{i}:=$ $\left\{y_{1}+a y_{2}: y_{1}, y_{2} \in Y_{i}\right\}$ is in definable bijection with $Y_{i} \times Y_{i}$ and of size less than $n_{i}$. By Theorem 26, all subsets of $T_{i}$, hence of $Y_{i} \times Y_{i}$, are uniformly definable by some $\psi_{2}(y, u)$. Similarly, we can show that all subsets of $Y_{i} \times Y_{i} \times Y_{i}$ are uniformly definable by some $\psi_{3}(y, u)$.

We may assume that all subsets of $Y_{i}$ (and $Y_{i} \times Y_{i}, Y_{i} \times Y_{i} \times Y_{i}$ ) can be defined uniformly by parameters in $\mathbb{F}_{p_{i}}{ }_{i}$. For $a \in \mathbb{F}_{p_{i} n_{i}}$, we write $S_{a}^{1} \subseteq Y_{i}$ for the set $\psi_{1}\left(a, \mathbb{F}_{p_{i}}\right)$ and $S_{a}^{2} \subseteq Y_{i} \times Y_{i}, S_{a}^{3} \subseteq Y_{i} \times Y_{i} \times Y_{i}$ for $\psi_{2}\left(a, \mathbb{F}_{p_{i}} n_{i}\right), \psi_{3}\left(a, \mathbb{F}_{p_{i}} n_{i}\right)$ respectively.

Now define a relation $R_{+} \subseteq\left(\mathbb{F}_{p_{i}} n_{i}\right)^{3}$ by: $R_{+}(a, b, c)$ if there exist $g \in \mathbb{F}_{p_{i} n_{i}}$ and $y \neq y^{\prime} \in Y_{i}$ such that

- either $S_{g}^{3}$ is the graph of a bijective function from $\left(S_{a}^{1} \times\{y\}\right) \cup\left(S_{b}^{1} \times\left\{y^{\prime}\right\}\right)$ to $S_{c}^{1}$;

- or $S_{c}^{1}=Y_{i}$ and $S_{g}^{3}$ is the graph of a surjective function from $\left(S_{a}^{1} \times\{y\}\right) \cup\left(S_{b}^{1} \times\left\{y^{\prime}\right\}\right)$ to $Y_{i}$;

Similarly, we define $R_{\times} \subseteq\left(\mathbb{F}_{p_{i} n_{i}}\right)^{3}$ by: $R_{\times}(a, b, c)$ if there exists $g \in \mathbb{F}_{p_{i}}^{n_{i}}$ such that

- either $S_{g}^{3}$ is the graph of a bijective function from $S_{a}^{1} \times S_{b}^{1}$ to $S_{c}^{1}$;

- or $S_{c}^{1}=Y_{i}$ and $S_{g}^{3}$ is the graph of a surjective function from $S_{a}^{1} \times S_{b}^{1}$ to $Y_{i}$;

We also define an equivalence relation $E \subseteq\left(\mathbb{F}_{p_{i}}\right)^{2}$ by: $E(a, b)$ if and only if there exists $g \in \mathbb{F}_{p_{i} n_{i}}$ such that $S_{g}^{2}$ is the graph of a bijective function from $S_{a}^{1}$ to $S_{b}^{1}$.

It is easy to see then that $R^{+}, R^{\times}$respect the equivalence relation $E$ and

$$
\left(\left|Y_{i}\right|,+, \times\right) \simeq\left(\left(\mathbb{F}_{p_{i}} n_{i}\right)^{2} / E, R^{+} / E, R^{\times} / E\right)
$$

Corollary 30. Let $(F$, Frob $) \in \mathcal{S}$ and $T:=T h(F$, Frob). Then $T$ has the strict order property and TP2. Moreover, $T$ is not decidable.

Proof. As the fixed field $\operatorname{Fix}(F):=\{x \in F: \sigma(x)=x\}$ is definable and satisfies the condition in Theorem 26, every pseudofinite subset of $\operatorname{Fix}(F)$ can be coded uniformly by some formula $\varphi(x, t)$. In particular, it will code some infinite strictly increasing chain $A_{1} \subsetneq A_{2} \subsetneq A_{3} \subsetneq \cdots$ of subsets of $\operatorname{Fix}(F)$. Therefore, $T$ has the strict order property.

Let $\varphi(x, t)$ be the same formula. To see that $T$ has TP2, by compactness, we only need to show that given any $n \in \mathbb{N}$, there is some $\left(a_{i j}\right)_{1 \leq i, j \leq n}$ such that for any $1 \leq i \leq n$, we have $\left\{\varphi\left(x, a_{i j}\right): 1 \leq j \leq n\right\}$ is 2 -inconsistent and $\left\{\varphi\left(x, a_{i f(i)}\right): 1 \leq i \leq n\right\}$ is consistent for any $f:\{1, \ldots, n\} \rightarrow\{1, \ldots, n\}$. 
Given $n \in \mathbb{N}$, let $A_{n} \subseteq \operatorname{Fix}(F)$ be a set with $n^{n}$-many elements. Fix a bijection $\eta$ : $A_{n} \rightarrow\{1, \ldots, n\}^{\{1, \ldots, n\}}$ where $\{1, \ldots, n\}^{\{1, \ldots, n\}}$ is the set of all functions from $\{1, \ldots, n\}$ to itself. Let $\left(a_{i j}\right)_{1 \leq i, j \leq n}$ be such that $\varphi\left(x, a_{i j}\right)$ codes the set

$$
B_{i j}:=\left\{a \in A_{n}: \eta(a)(i)=j\right\} \subseteq A_{n} .
$$

For any $1 \leq i \leq n$, as $B_{i 1}, \ldots, B_{i n}$ form a complete partition of $A_{n}$, we get $\left\{\varphi\left(x, a_{i j}\right)\right.$ : $1 \leq j \leq n\}$ is 2 -inconsistent. On the other hand, for any $f:\{1, \ldots, n\} \rightarrow\{1, \ldots, n\}$ the element $\eta^{-1}(f) \in A_{n}$ witnesses that $\left\{\varphi\left(x, a_{i f(i)}\right): 1 \leq i \leq n\right\}$ is consistent.

As $(F$, Frob) interprets ultraproducts of initial segments of natural numbers with truncated addition and multiplication by Corollary 30, the undecidability follows from [10, Section 4].

The following part concerns the algebraic closure in $(F$, Frob $) \in \mathcal{S}$. Let $F$ be a pseudofinite field and $F^{a l g}$ be the smallest algebraically closed field containing $F$. Take a tuple $a \in F$. Then the algebraic closure in the pseudofinite field $\operatorname{acl}_{F}(a)$ is simply the algebraic closure in $F^{a l g}$ intersected with $F$, i.e., $\operatorname{acl}_{F}(a)=\operatorname{acl}_{F a l g}(a) \cap F$.

As ACFA is the model companion of the theory of difference fields, we can embed $(F$, Frob) into some $(K, \sigma) \models A C F A$. We might wonder if similarly, the algebraic closure in the theory of $(F$, Frob) is the same as the algebraic closure in $(K, \sigma)$ intersected with $F$. But the answer is negative. In fact, we have the following.

Lemma 31. For any $n>0$, there is some $(F$, Frob $) \in \mathcal{S}$ and element $a_{n} \in F$ such that $a_{n}$ is in the definable closure of tuple $b_{n}$ in $(F$, Frob $)$, but $\operatorname{deg}_{\sigma}\left(a_{n} / b_{n}\right)=n$.

We need a small lemma first.

Lemma 32. Let

$$
\varphi\left(x ; y_{1}, \cdots, y_{n}\right):=\exists z\left(z^{2}=x+y_{1}\right) \wedge \bigwedge_{2 \leq i \leq n} \forall z \neg\left(z^{2}=x+y_{i}\right) .
$$

There is $C_{n} \in \mathbb{R}$ such that for any $\mathbb{F}_{q}$ with $\operatorname{char}\left(\mathbb{F}_{q}\right) \neq 2$ and $b_{1}, \cdots, b_{n}$ distinct $n$ elements in $\mathbb{F}_{q}$, we have

$$
|| \varphi\left(\mathbb{F}_{q}, b_{1}, \cdots, b_{n}\right)\left|-\frac{q}{2^{n}}\right| \leq C_{n} \cdot q^{\frac{1}{2}} .
$$

Proof. Given distinct elements $b_{1}, \cdots, b_{n} \in \mathbb{F}_{q}$. Take an element $a \in \mathbb{F}_{q}$ such that $b$ is not a square. Let $J$ be the ideal in $\mathbb{F}_{q}\left[X, X_{1}, \cdots, X_{n}\right]$ generated by

$$
\left\{X_{1}^{2}-\left(X+b_{1}\right)\right\} \cup\left\{X_{i}^{2}-a\left(X+b_{i}\right): 2 \leq i \leq n\right\} .
$$

By Fact 24, $J$ is absolutely prime, whence $V(J)$ is an absolutely irreducible variety. By the Lang-weil estimate

$$
|| V(J) \cap\left(\mathbb{F}_{q}\right)^{n+1}-q \mid \leq N_{n} \cdot q^{\frac{1}{2}},
$$

where $N_{n}$ is a constant only depends on the degree and dimension of the variety, which in our case is independent with $b_{1}, \cdots, b_{n}, a$ and $\mathbb{F}_{q}$ and only depends on $n$. Let

$$
\pi: V(J) \cap\left(\mathbb{F}_{q}\right)^{n+1} \rightarrow \mathbb{F}_{q}
$$


be the projection on the the first coordinate. Clearly, $\pi$ is a $2^{n}$-to-one function. Therefore,

$$
\left|\varphi\left(\mathbb{F}_{q}, b_{1}, \cdots, b_{n}\right)\right|=\left|\pi\left(V(J) \cap\left(\mathbb{F}_{q}\right)^{n+1}\right)\right|=\frac{1}{2^{n}} \cdot\left|V(J) \cap\left(\mathbb{F}_{q}\right)^{n+1}\right| .
$$

Let $C_{n}:=\frac{N_{n}}{2^{n}}$. We conclude that

$$
|| \varphi\left(\mathbb{F}_{q}, b_{1}, \cdots, b_{n}\right)\left|-\frac{q}{2^{n}}\right| \leq C_{n} \cdot q^{\frac{1}{2}} .
$$

Now we prove Lemma 31.

Proof. Given $n \in \mathbb{N}$, for each $p \in \mathbb{P}$, let $k_{p} \in \mathbb{N}$ be such that

- $k_{p}>\max \left\{f(p, p), 14 p^{n}\right\}$ where $f(p, p)$ is given by Equation 1;

- $n$ ! divides $k_{p}$;

- $\frac{p^{k_{p}}}{2^{p^{n}}}>2 C_{p^{n}} \cdot p^{\frac{k_{p}}{2}}$.

Let $(F$, Frob $):=\prod_{p \in \mathbb{P}}\left(\mathbb{F}_{p^{k_{p}}}\right.$, Frob $\left._{p}\right) / \mathcal{U}$ where $\mathcal{U}$ is a non-principal ultrafilter on $\mathbb{P}$. Clearly, $(F$, Frob $) \in \mathcal{S}$ and $\operatorname{Fix}\left(\sigma^{n}\right):=\left\{x \in F: \sigma^{n}(x)=x\right\} \neq \operatorname{Fix}\left(\sigma^{k}\right)$ for any $k<n$.

Take an element $a_{n} \in \operatorname{Fix}\left(\sigma^{n}\right)$ such that $\operatorname{deg}_{\sigma}\left(a_{n}\right)=n$. Let

$$
\xi\left(x, a_{n}\right):=\exists z\left(z^{2}=a_{n}+x\right) \wedge \forall y\left(\sigma^{n}(y)=y \wedge\left(y \neq a_{n} \rightarrow \neg \exists z\left(z^{2}=y+x\right)\right)\right) .
$$

As $p>14 p^{n}$ for each $p \in \mathbb{N}$, by Theorem 26 and the subsequent remark, we know that $Y_{n}:=\xi\left((F\right.$, Frob $\left.), a_{n}\right) \neq \emptyset$. We claim that $\boldsymbol{\delta}_{F}\left(Y_{n}\right)=1$. Suppose $a_{n}=\left(a_{p}\right)_{p \in \mathbb{P}} / \mathcal{U}$. For each $p \in \mathbb{P}$, let $a_{p}, b_{1}, \cdots, b_{p^{n}-1}$ be a list of all elements in $\mathbb{F}_{p^{n}} \subseteq \mathbb{F}_{p^{k_{p}}}$. Let

$$
\varphi\left(x, y_{1}, \cdots, y_{p^{n}}\right):=\exists z\left(z^{2}=x+y_{1}\right) \wedge \bigwedge_{2 \leq i \leq p^{n}} \forall z \neg\left(z^{2}=x+y_{i}\right) .
$$

Note that for any $b \in \mathbb{F}_{p^{k_{p}}}$ we have

$$
\xi\left(\left(\mathbb{F}_{p^{k_{p}}}, \operatorname{Frob}_{p}\right), a_{p}\right)=\varphi\left(\mathbb{F}_{p^{k+p}}, a_{p}, b_{1}, \cdots, b_{p^{n}-1}\right) .
$$

By Lemma 32,

$$
|| \varphi\left(\mathbb{F}_{p^{k+p}}, a_{p}, b_{1}, \cdots, b_{p^{n}-1}\right)\left|-\frac{p^{k_{p}}}{2^{p^{n}}}\right| \leq C_{p^{n}} \cdot p^{\frac{p^{k_{p}}}{2}},
$$

for all $p>2$. Therefore,

$$
\left|Y_{n}\right| \geq \frac{p^{k_{p}}}{2^{p^{n}}}-C_{p^{n}} \cdot p^{\frac{p^{k_{p}}}{2}}>\frac{1}{2} \cdot \frac{p^{k_{p}}}{2^{p^{n}}} .
$$

Since

$$
\lim _{p \rightarrow \infty} \frac{\log \left(p^{k_{p}} / 2 \cdot 2^{p^{n}}\right)}{\log p^{k_{p}}}=1
$$

we get $\boldsymbol{\delta}_{F}\left(Y_{n}\right)=1$. 
Take an element $b_{n} \in Y_{n}$ such that $\boldsymbol{\delta}_{F}\left(b_{n}\right)>0$. Note that $a_{n} \in \operatorname{dcl}\left(b_{n}\right)$ and $\boldsymbol{\delta}_{F}\left(a_{n}\right)=0$. Thus,

$$
\boldsymbol{\delta}_{F}\left(b_{n} / a_{n}\right)=\boldsymbol{\delta}_{F}\left(a_{n}, b_{n}\right)-\boldsymbol{\delta}_{F}\left(a_{n}\right)=\boldsymbol{\delta}_{F}\left(b_{n}\right)+\boldsymbol{\delta}_{F}\left(a_{n} / b_{n}\right)-\boldsymbol{\delta}_{F}\left(a_{n}\right)=\boldsymbol{\delta}_{F}\left(b_{n}\right)>0 .
$$

Therefore, $S U_{A C F A}\left(b_{n} / a_{n}\right)=\omega$. By our choice, we also have $S U_{A C F A}\left(b_{n}\right)=\omega$. Hence, $a_{n}$ is independent with $b_{n}$ in $\left(\tilde{F}\right.$, Frob). Again, by our choice, $\operatorname{deg}_{\sigma}\left(a_{n}\right)=n$. But if $\operatorname{deg}_{\sigma}\left(a_{n} / b_{n}\right)<n$, then $a_{n}$ and $b_{n}$ will not be independent in $(\tilde{F}$, Frob) in the theory of ACFA. We conclude that $\operatorname{deg}_{\sigma}\left(a_{n} / b_{n}\right)=n$ and $a_{n}$ is in the definable closure of $b_{n}$.

\section{References}

[1] James Ax. The elementary theory of finite fields. Annals of Mathematics, pages 239-271, 1968.

[2] Antonio Cafure and Guillermo Matera. Improved explicit estimates on the number of solutions of equations over a finite field. Finite Fields and Their Applications, 12(2):155-185, 2006.

[3] Zoé Chatzidakis. Model theory of difference fields. In The Notre Dame Lectures, P. Cholak, series editor, Lecture notes in Logic, pages 45-96. A.K. Peters, 2005.

[4] Zoé Chatzidakis and Ehud Hrushovski. Model theory of difference fields. Transactions of the American Mathematical Society, 351(8):2997-3071, 1999.

[5] Zoé Chatzidakis, Ehud Hrushovski, and Ya'acov Peterzil. Model theory of difference fields, ii: Periodic ideals and the trichotomy in all characteristics. Proceedings of the London Mathematical Society, 85(2):257-311, 2002.

[6] Zoé Chatzidakis, Lou van den Dries, and Angus Macintyre. Definable sets over finite fields. J. reine angew. Math, 427:107-135, 1992.

[7] Jean-Louis Duret. Les corps faiblement algébriquement clos non séparablement clos ont la propriété dindépendance. In Model theory of algebra and arithmetic, pages 136-162. Springer, 1980.

[8] Ehud Hrushovski et al. On pseudo-finite dimensions. Notre Dame Journal of Formal Logic, 54(3-4):463-495, 2013.

[9] Ehud Hrushovski and Anand Pillay. Groups definable in local fields and pseudofinite fields. Israel Journal of Mathematics, 85(1):203-262, 1994.

[10] Michał Krynicki and Konrad Zdanowski. Theories of arithmetics in finite models. The Journal of Symbolic Logic, 70(1):1-28, 2005.

[11] Mark Ryten and Ivan Tomašić. Acfa and measurability. Selecta Mathematica, New Series, 11(3):523-537, 2006.

[12] Mark Jonathan Ryten. Model theory of finite difference fields and simple groups. PhD thesis, Citeseer, 2007. 